\title{
Restating the self-thinning rule
}

\author{
from Peter D. Moore
}

MORTALITY amongst young organisms is one of the basic observations which led Darwin to propound his theory of natural selection. Often this mortality results from the competitive stresses which are derived from a high density of other members of the same species. Among plants the process is often referred to as self thinning. The nature of this process was analysed by Yoda et al. (J. Biol. Osaka City Univ. 14, $107 ; 1963)$ in pure even-aged populations of plants under density stress. Their most important finding was that if one plots the log of mean individual plant weight against the log of surviving plant density one obtains a straight line with a slope of $-3 / 2$.

The self-thinning rule which emerges from these observations can be summarised by the formula,

$$
W=K p^{-3 / 2}
$$

where $W=$ mean plant weight and $p=$ density.

The rule was examined by White and Harper (J. Ecol. 58, 467; 1970), who provided new experimental data and who also analysed a body of published data most of which agreed with the observations of Yoda et al. Variations in nutrient availability had no influence upon the slope of the line, but did affect its position on the axes. Fertility resulted in a higher degree of thinning. On examining published data concerning light intensity variations and thinning in Helianthus annuus, however, they found that decreasing light intensity was correlated with less steep slopes in the log plant weight/log density lines.

Kays and Harper (J. Ecol. 62, 97; 1974) have since experimented with the rye grass, Lolium perenne and have tested the effect of light intensity upon both individual (genet) and tiller density and weight. At high light intensities genet weight and density obeyed the $3 / 2$ thinning law, but at the low light levels the slope fell to 1 . On this basis they suggested that it is the reduced light intensity within a sward which causes the stress, resulting in mortality.

In this issue of Nature (page 330, Westoby re-examines the $3 / 2$ selfthinning law in the context of work on the influence of light on leaf mor- phology. It is known that leaves produced under low light intensity have a larger area, but not normally a greater weight, than those produced in high light levels. Area : weight ratios are therefore raised at low light. This being so, Westoby has re-examined the original Helianthus annuus data analysed by White and Harper, but has plotted mean leaf area on a log scale rather than log mean plant weight. The result is a line with a $3 / 2$ slope which is independent of the light intensity at which the data was obtained. Thus he reformulates the self-thinning rule as

$$
L=K p^{-3 / 2}
$$

where $L=$ leaf area. Not only is this formula of more general application than the original statement of the rule, it also permits one to predict mortality rates given information on the changes in leaf area index (leaf area: ground area) of a population in time. Such information is already available for many crop and forage species, hence one can work from this data to a better understanding of their population dynamics under density stress.

\section{Persistent infections without proviral DNA}

from Rohin Weiss

IN 1975, Zhdanov (Nature 256, 471) and Simpson and linuma (Proc. natn. Acad. Sci. U.S.A. 72, 3230) claimed to have found integrated DNA copies of RNA viruses such as sindbis, measles and respiratory syncytial viruses in the cells of persistently infected cultures. They suggested that the formation of DNA 'proviruses' might lead to the persistant state. Recently, John Holland and associates (Holland et al., J. gen. Virol., 33, 193; 1976) have concluded from a series of meticulous experiments on long-term persistent cultures of $\mathrm{BHK}$ 21 cells infected with vesicular stomatitis virus (VSV) that there is no evidence for 'proviral' DNA copies of the VSV genome in those cultures. Hybridisation tests capable of detecting 1 viral genome per 40 cells (or 1/40 of a genome per cell) gave no indication of sequences homologous to viral RNA in the DNA of persistently infected cells, and DNA transfection attempts also failed to reveal any biological activity. Transfection with DNA was also negative in a variety of other cultures persistently infected with rabies. influenza, measles, mumps or lymphocytic choriomeningitis viruses. Thus it is clear that the formation of proviral DNA is by no means a prerequisite for the establishment of persistent infections with negative strand RNA viruses.

Holland and Villarreal originally established the persistently infected VSV carrier culture (Proc. natn. Acad. Sci. U.S.A. 71, 2956; 1974) by using a temperature-sensitive mutant ( $t s \mathrm{G} 31$ ) together with an excess of defective interfering (DI) particles. Nucleocapsids of the DI particles persist in the carrier cultures and presumably interfere with complete particle replication. Nevertheless, most cells in the carrier cultures continue to synthesise viral RNA (Villarreal and Holland, $J$, gen. Virol. 33, 213; 1976) and proteins even after more than 2 years in culture. Cell cloning can, however, yield 'cured' cell populations lacking all evidence of infection.

Holland's group's studies support the view put forward by Huang and Baltimore in 1970 (Nature 226, 325-327) that defective interfering particles have a role in viral disease processes. Doyle and Holland (Proc. natn. Acad. Sci. U.S.A. 70, 2105; 1973) had previously shown that the addition of excess DI particles to biologically active VSV protected mice against early lethal intracerebral infection. But DI particles might lead to 'slow' persistent infection, as opposed to acute infection, in vivo too, and with some viruses (measles, for example) and some hosts (such as man) the consequences to the host may be far more dire than acute infection. Since DI particles depend on non-defective virus for replication, but then replicate to excess and interfere with the non-defective virus, cyclic production of mature and DI virus may ensue, as Palma and Huang ( $J$. infect. Dis. 129,402 ; 1974) have shown for VSV when fresh cells are added to infected cultures. The mechanism of interference by DI particles is not clear, but they may compete for polymerase. DI particles typically contain a part of the viral genome as the usual negative strand RNA, although Lazzarini et al. (J. molec. Biol. 97, 289; 1975) have described interesting covalently-linked 